Cronfa - Swansea University Open Access Repository

This is an author produced version of a paper published in :

Applied Surface Science

Cronfa URL for this paper:

http://cronfa.swan.ac.uk/Record/cronfa5734

http://dx.doi.org/10.1016/j.apsusc.2010.03.089

This article is brought to you by Swansea University. Any person downloading material is agreeing to abide by the terms of the repository licence. Authors are personally responsible for adhering to publisher restrictions or conditions. When uploading content they are required to comply with their publisher agreement and the SHERPA RoMEO database to judge whether or not it is copyright safe to add this version of the paper to this repository. http://www.swansea.ac.uk/iss/researchsupport/cronfa-support/ 


\title{
Surface defects in semiconductor lasers studied with cross-sectional scanning tunneling microscopy
}

\author{
R. J. Cobley*, K. S. Teng, M. R. Brown, P. Rees and S. P. Wilks \\ Multidisciplinary Nanotechnology Centre, School of Engineering, Singleton Park, Swansea, SA1 4HZ, UK.
}

Keywords: Scanning tunneling microscopy (STM); semiconductor laser; passivation; AlGaAs; InP PACS: 07.79.Cz; 68.37.Ef; 68.37.Yz; 73.20.Fz

\begin{abstract}
Cross-sectional scanning tunneling microscopy is used to study defects on the surface of semiconductor laser devices. Step defects across the active region caused by the cleave process are identified. Curved blocking layers used in buried heterostructure lasers are shown to induce strain in the layers above them. Devices are also studied whilst powered to look at how the devices change during operation, with a numerical model that confirms the observed behavior. Whilst powered, low-doped blocking layers adjacent to the active region are found to change in real time, with dopant diffusion and the formation of surface states. A tunneling model which allows the inclusion of surface states and tip induced band bending is applied to analyze the effects on the tunneling current, confirming that the doping concentration is reducing and defect surface states are being formed.

* Corresponding author: richard.j.cobley@swansea.ac.uk
\end{abstract}

This is the accepted version of the following article:

Applied Surface Science 256, 5736-5739 (2010)

Doi: 10.1016/j.apsusc.2010.03.089

For which the copyright is held by Elevier. It has been published in final form at: http://www.sciencedirect.com/science/article/pii/S0169433210003806 


\section{Introduction}

Cross-sectional scanning tunneling microscopy (X-STM) on the unreconstructed (110) surface of semiconductor lasers gives a view across all growth layers in the devices. For defect free surfaces there are no mid band gap surface states and the technique can be used to study surface morphology, cleave-induced defects, doping concentration, interface quality, atomic-level intermixing, potentiometry and surface passivation, amongst others ${ }^{1,2,3,4,5}$.

Semiconductor lasers have a wide range of uses from optical communications to data storage ${ }^{6}$. Typical surface defects formed during production of these devices include steps, strain-induced changes, lattice disordering, intermixing at heterojunctions and dopant diffusion. These defects change the material properties and introduce mid band gap surface states which detrimentally affect device performance and ultimately lead to device failure ${ }^{7}$. Surfaces of such optoelectronic devices are coated with layers of material which passivate these surface states by moving their bond energies out of the band gap ${ }^{8,9,10}$.

Here X-STM is applied to the unpassivated (110) facet of semiconductor lasers to study a range surface defects which affect device operation. Using a technique which allows devices to be powered whilst performing X-STM, devices are also studied while operating in real time, to look at defect generation and evolution. $^{11,12}$. This paper will look first at unpowered devices at rest to identify surface steps and strain. It will then look at performing X-STM on biased devices and the repeatable and reversible changes that occur in devices when studied in this way, along with a model which predicts the same changes. Finally lowdoped regions near the active layers are found to undergo irreversible damage whilst under operation, due to dopant out-diffusion and surface defect state formation. The process is studied and modeled.

\section{Experimental}

Samples are cleaved in situ in a commercial ultra high vacuum system to expose a clean facet for investigation. Tips are made from electrochemically etched tungsten wire, cleaned in situ using high voltage pulses. The system has been modified to allow a potential to be dropped across the device being studied, 
independently of the applied gap voltage between the same sample and tip, all relative to a common ground point at one of the sample contacts. This allows the device to be powered whilst the scan continues.

Two types of laser device are used in this investigation: an AlGaAs/InGaAs double quantum well (DQW) structure, and an InP/InGaAsP buried heterostructure laser (BHL). More detailed information on the experimental procedure and device properties is given elsewhere ${ }^{11,13}$.

\section{Numerical Model}

Two models are used to analyze the data which will be presented. The first uses a method based on Duke, as implemented by Feenstra1 ${ }^{14}$. A planar tunneling calculation is performed with no band bending in the sample surface, as a summation over all incoming states in $k$-space, with the calculated transmission factor for each state ${ }^{11}$. The full E- $k$ bandstructure for each material is calculated using a local pseudopotential method based on that of Cohen and Bergstresser, implemented by Fischetti and Laux ${ }^{15,16}$. Energy diagrams for the devices under test were generated using a drift-diffusion model which fully coupled the electron and hole equations with Poisson's equation ${ }^{17}$. This gave the band profiles for both the sample at rest and showed how the devices behaved under bias.

A second tunneling model was used to more accurately study areas in the device which changed during operation. In low-doped materials the electrostatic potential of the scanning tip can induce depletion or accumulation layers in the sample being studied. Some of the applied potential between the sample and tip is dropped across this depletion region in which the sample bands bend. Here we use a second tunneling model by Feenstra which allows band bending at the surface and the inclusion of the discrete surface states formed by this potential variation. The profile of the tip-induced band bending is calculated by using Feenstra's SEMITIP3 which also allows the inclusion of surface defect states ${ }^{18,19,20,21,22}$.

\section{Results and discussion}

The process of cleaving the laser wafer, used both in industry and research, can leave step defects across the facet, as shown in figure 1(a) for the BHL. Here, two levels of jagged step depression are visible across the 
otherwise atomically flat active region. The total measured depth is around $0.608 \mathrm{~nm}$, slightly larger than the lattice constant of 0.587 due to surface relaxation ${ }^{23}$.

Figure 1(b) shows a larger image of the active region of the same device, with a gradient from the top of the image down towards the active region, culminating in depressions visible above the curved blocking layers. A plot along the marked line in that image is shown in figure 1(c). Shear force topographic images of the same device have shown that the origin of this gradient is not a physical height difference and therefore it must be an electronic effect ${ }^{24}$. Spectroscopy in this region confirms that the area is undergoing tensile strain5. The layers grown above the curved blocking layer are strained and relax out towards the surface producing the electronic effect visible with STM. Understanding how complicated confinement structures alter the material parameters and therefore device operation is crucial to optimizing these new devices.

Next we turn our attention to studying these devices while they are powered and active. Figure 2 shows images of the DQW laser at rest and biased, and Figure 3 the BHL. Electronic gradients exist across images due both to the change in contrast as the tip scans over the differently doped regions and materials, and because the sample is always physically tilted at this scale. As a bias is applied to the sample these electronic gradients, and the relative contrast of features in the image, change. However, the physical topography does not change and we can look at how a scan changes electronically simply by subtracting the image at bias from the image at rest. The STM images and line plots in Figure 2 (a) - (c) and Figure 3 have had these gradients removed to show relative height. Figure 2 (d) and (e) however, show the change in this gradient across the image as the height change over the two micrometer length off the image (solid line), and overlay on top the change expected from the first model presented earlier (dashed line).

Electronic gradients and the relative contrast of features, such as the quantum well heights, are found to be reversible. The contrast in the image changes as power is applied to the device and then reduces back to the starting point when power is removed from the device. The magnitude of the change of gradients and features across an image is also consistent with the model. As seen in figure 2 (d), at higher biases the 
model predicts slightly less change in the gradient due to model limitations in handling the optical behavior of the laser cavity.

With this standard pattern of behavior established, it is then possible to look at changes that occur on the surface of devices when they are left to run over a long period of time, or driven with higher currents ${ }^{12}$. One such change occurs in the low-doped regions that surround the quantum wells. An example for the BHL is shown in figure 4. The purpose of these low-doped layers is to prevent dopants in the higher-doped layers from diffusing in to the nominally undoped array of quantum wells and barriers. However in both devices these low-doped layers undergo permanent modification during operation. For the example in figure 4 when tunneling in to the BHL the p-type buffer appears brighter after modification whereas the n-type buffer appears darker.

This modification cannot be explained by a physical surface change, or by the formation of dark-area defects, because one layer increases in contrast as another reduces. Also when imaged with the opposite polarity gap voltage the contrast change in the buffer layers goes the other way. It must therefore be an electronic change at the surface that shifts the Fermi level back towards intrinsic, which matches the direction of contrast change in all samples studied. Two effects can account for such a modification; a reduction in the doping concentration and the formation of surface states.

Since these layers are less doped, their conductivity is lower than the surrounding layers and more of applied device voltage would be dropped across them, causing them to heat more. Localized heating could account for dopant out-diffusion from these layers. Also, low-temperature heating of III-V surfaces is known to cause evaporation primarily of the anion species leading to anion-vacancy defects, which for InP and GaAs materials generate mid- band gap surface states ${ }^{25}$. These additional states can pin the Fermi level at the surface and cause the bands to bend back towards mid gap.

In order to look at the relative influence of these two effects on the STM process, the second model is applied to the change seen in both the DQW laser and the BHL. For this, we start with an isolated layer of 
AlGaAs or InP doped as per the growth specifications to $2 \mathrm{e}^{-17 \mathrm{~cm}^{-3}}$, assuming no surface states. In these layers, and especially as the doping concentration is reduced, the tip-induced band bending effect at the surface becomes important. For every point in the calculation Feesntra's SEMITIP3 program is used to establish the effect of the tip's electrostatic behavior in modifying the surface band bending of the semiconductor. This result is then used in the second tunneling model discussed earlier to calculate the tunneling current for that condition. A set point is established by varying the area of the tip in the calculation until the tunneling current matches that from the experiment. Once this condition is established, the doping concentration is incrementally modified and a feedback routine modifies the tip height until the same tunneling current is reached. The deflection in the tip z-height required to reach the same tunneling current is recorded, which mimics the information recorded from the STM process. The density of surface states is then increased in the same way to again analyze the change in tip height. A uniform distribution of surface states is used, with the charge neutrality level at mid gap. There are several free parameters in this model and more detail is given elsewhere ${ }^{22 .}$

An example of the modeled change of the tip height is given in figure 5. As the doping concentration reduces (right to left, circles) the model confirms a reduction in the contrast of $\Delta \mathrm{z}=-130.0 \mathrm{pm}$ at $1 \mathrm{e} 15 \mathrm{~cm}^{-3}$. As the surface state density increases (left to right, crosses) a contrast reduction is again predicted, down to $\Delta \mathrm{z}=-193.2 \mathrm{pm}$ at $1 \mathrm{e} 10 \mathrm{~cm}^{-2}$. The experimentally measured contrast change was $\Delta \mathrm{z}=-121.3 \mathrm{pm}$. For this example both the doping concentration reduction and the formation of surface states could be the origin of the observed change in the STM image - the direction of the change and the magnitude matches between modeling and experiment. In other layers or with different tunneling conditions, STM can be shown through the model to be sensitive to just one of the processes. In all these conditions a change is still observed in the low doped layers, which confirms both processes are occurring on the surface of the device.

\section{Summary}

X-STM has been applied to the surface of III-V optoelectronics devices to study surface defects that occur on experimental and commercial devices. It can detect and quantify physical step defects on the surface and strain induced surface changes. Devices can be studied at bias in order to measure the in-operation 
response, with repeatable behavior which matches modeling. Low doped layers adjacent to the active region are found to irreversibly change under operation. A tunneling model which looks at the change in doping concentration and the formation of surface defect states concludes that the change observed with STM is due both to the reduction of doping concentration and the formation of surface defect states in those layers. This will be accompanied with likely out-diffusion in to neighboring layers, and increased heating, leading to the failure of the device.

\section{Acknowledgements}

This work was funded through a Royal Academy of Engineering (UK) research fellowship for RJC and through the Engineering and Physical Sciences Research Council (UK).

\section{References}

\footnotetext{
${ }^{1}$ R. M. Feenstra, Physica B 274 (1999) 796.

${ }^{2}$ R. M. Feenstra, E. T. Yu, J. M. Woodall, P. D. Kirchner, C. L. Lin and G. D. Pettit, Appl. Phys. Lett. 61 (1992) 795.

${ }^{3}$ H. J. Chen, H. A. McKay, R. M. Feenstra, G. C. Aers, P. J. Poole, R. L. Williams, S. Charbonneau, P. G.
} Piva, T. W. Simpson and I. V. Mitchell, J. Appl. Phys. 89 (2001) 4815.

${ }^{4}$ P. Muralt, H. Meier, D. W. Pohl and H. W. M. Salemink, Appl. Phys. Lett. 50 (1987) 1352.

${ }^{5}$ R. J. Cobley, K. S. Teng, T. G. G. Maffeïs and S. P. Wilks, Surf. Sci. 600 (2006) 2857.

${ }^{6}$ E. Kapon, Semiconductor Lasers 1: Fundamentals (Academic Press, London, 1999).

${ }^{7}$ O. Ueda, J. Electrochem. Soc.: Rev. News, 135 (1988) $11 \mathrm{C}$.

${ }^{8}$ K. S. Teng, P. R. Dunstan, S. P. Wilks and R. H. Williams, Appl. Phys. Lett. 75 (1999) 2590.

${ }^{9}$ S. P. Wilks, K. S. Teng, P. R. Dustan and R. H. Williams, Appl. Surf. Sci. 190(2002) 467.

${ }^{10}$ H. Hasegawa and M. Akazawa, App. Surf. Sci. 255 (2008) 628.

${ }^{11}$ R. J. Cobley, K. S. Teng, M. R. Brown and S. P. Wilks, J. Appl. Phys. 102 (2007) 024306.

${ }^{12}$ R. J. Cobley, K. S. Teng, M. R. Brown, S. P. Wilks and P. Rees, Appl. Phys. Lett. 91 (2007) 081119.

${ }^{13}$ R. J. Cobley, K. S. Teng, M. R. Brown, T. G. G. Maffeïs and S. P. Wilks, Int. J. Nanoscience 3 (2004) 525.

${ }^{14}$ C. Duke, Tunneling in Solids (Academic Press, New York, 1969).

${ }^{15}$ M. L. Cohen and T. K. Bergstresser, Phys. Rev. 141 (1966) 789.

${ }^{16}$ M. V. Fischetti and S. E. Laux, IEEE Trans. on Elec. Dev. 38 (1991) 650.

${ }^{17}$ M. R. Brown, R. J. Cobley, K. S. Teng, P. Rees, S. P. Wilks, A. Sobiesierski, P. M. Smowton and P. Blood, J. Appl. Phys. 100 (2006) 084509.

${ }^{18}$ R. M. Feesntra, Y. Dong, M. P. Semtsiv and W. T. Masselink, Nanotech. 18 (2007) 044015.

${ }^{19}$ R. M. Feenstra, J. Vac. Sci. Technol. B 21 (2003) 2080.

${ }^{20}$ R. M. Feenstra, S. Gaan, G. Meyer and K. H. Rieder, Phys. Rev. B 71 (2005) 125316.

${ }^{21}$ Y. Dong, R. M. Feenstra, M. P. Semtsiv and W. T. Masselink, J. Appl. Phys. 103 (2008) 073704.

${ }^{22}$ R. J. Cobley, P. Rees, K. S. Teng and S. P. Wilks, “Analyzing real-time surface modification of operating semiconductor lasers using cross-sectional scanning tunneling microscopy", in preparation. 
${ }^{23}$ Handbook series on semiconductor parameters, Ed. M. levinshtein, S. Rumyantsev and M. Shur, World Scientific, Singapore (1996).

${ }^{24}$ M. P. Ackland, P. R. Dunstan and P. Rees, J. Microsc. 220 (2005) 229.

${ }^{25} \mathrm{Ph}$. Ebert, Surf. Sci. Rep. 33 (1999) 121. 


\section{Figures}

Figure 1:

STM images of the buried heterostructure laser (a) $+2.2 \mathrm{~V}$ gap voltage, $0.3 \mathrm{nA}$, showing cleave-induced steps across the active region, (b) $+2 \mathrm{~V}$ gap voltage, $0.5 \mathrm{nA}$ with (c) the z-profile along the white line shown from A to $\mathrm{C}$.
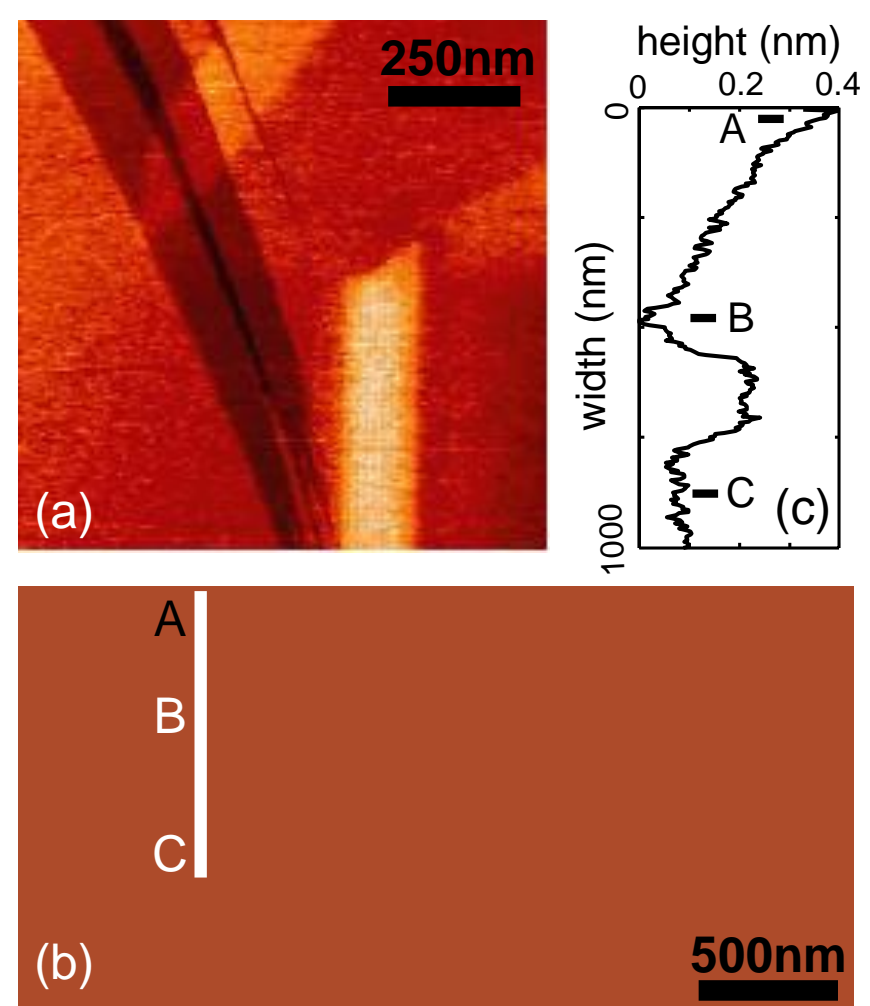
Figure 2:

STM images of the DQW device imaged with a gap voltage of $\mathrm{V}_{\mathrm{gap}}=-3.5 \mathrm{~V}, \mathrm{I}=0.4 \mathrm{nA}$. The sample is forward biased with (a) $\mathrm{V}_{\text {bias }}=0 \mathrm{~V}, \mathrm{I}=0 \mathrm{~mA}$ and (b) $\mathrm{V}_{\text {bias }}=0.98 \mathrm{~V}, \mathrm{I}=15 \mathrm{~mA}$, both plotted on the same $\mathrm{z}$-scale. Averaged line plots across the image are shown in (c). The change in the STM z-height from the extreme left of the image to the extreme right as a function of sample forward bias is shown in (d) for $\mathrm{V}_{\text {gap }}=-3 \mathrm{~V} \mathrm{I}=0.5 \mathrm{nA}$ and in (e) for $\mathrm{V}_{\mathrm{gap}}=+3 \mathrm{~V}, \mathrm{I}=1.0 \mathrm{nA}$ where the solid lines are the experimentally measured values and the dotted lines are the modeled response.
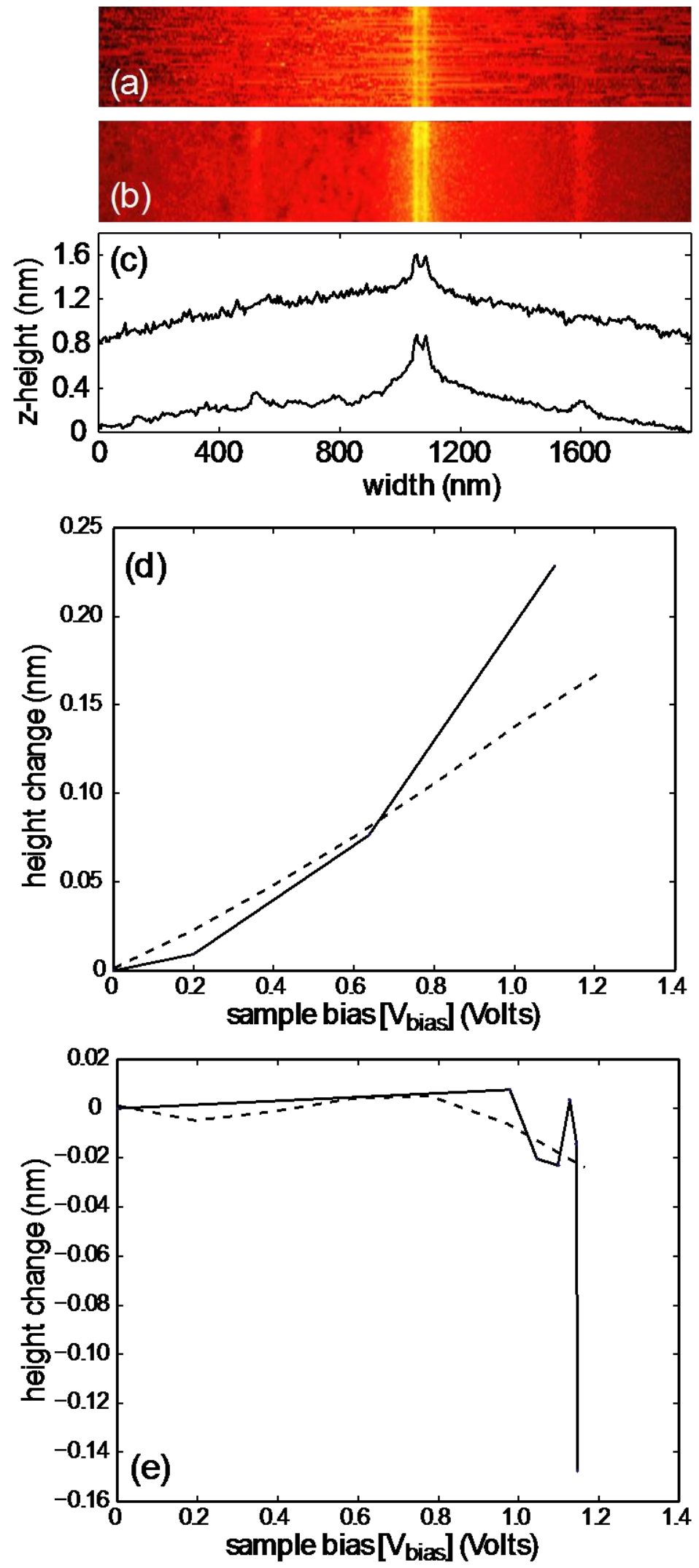
Figure 3:

STM images of the BHL device imaged at (a) $\mathrm{V}_{\text {gap }}=+1.89 \mathrm{~V}, \mathrm{I}=0.539 \mathrm{nA}$ and (b) $\mathrm{V}_{\text {gap }}=+1.89 \mathrm{~V}, \mathrm{I}=0.382 \mathrm{nA}$. The samples are biased at (a) $V_{\text {bias }}=0 \mathrm{~V}, \mathrm{I}=0 \mathrm{~mA}$ and (b) $\mathrm{V}_{\text {bias }}=0.9 \mathrm{~V}, \mathrm{I}=53 \mathrm{~mA}$. Noise spikes have been removed from the images, and both are plotted on the same z-scale with averaged line plots along the dotted white lines stacked with the $0 \mathrm{~V} \mathrm{~V}_{\text {bias }}$ case on top. The four diagonal stripes in each image are cleave-induced step defects and are not part of the discussion here.
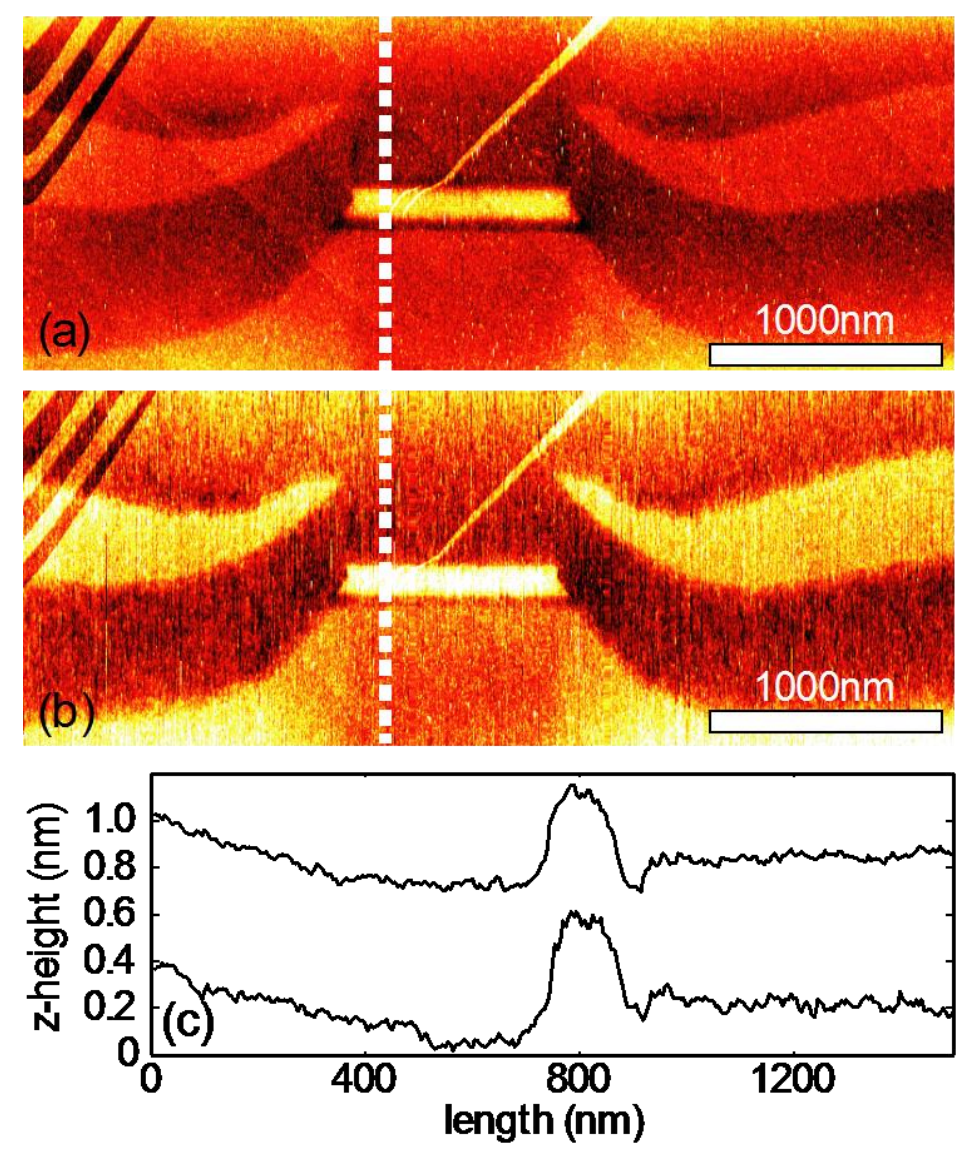
Figure 4:

STM images of the buried heterostructure laser with an STM gap voltage and tunneling current of $+2 \mathrm{~V}$ and $0.8 \mathrm{nA}$ with (a) a corresponding schematic. The current through the sample is (b) 0mA (before modification) and (c) $35 \mathrm{~mA}$ (during modification). (d) shows traces of fifteen averaged horizontal lines across the scan for $0 \mathrm{~A}$ before modification (solid), 35mA during modification (dashed) and $0 \mathrm{~A}$ after modification (dotted).

Neither the images nor the line plots have been processed or smoothed.

(a)

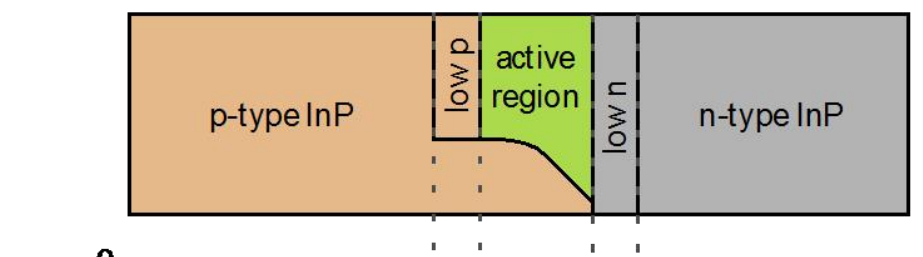

(b)

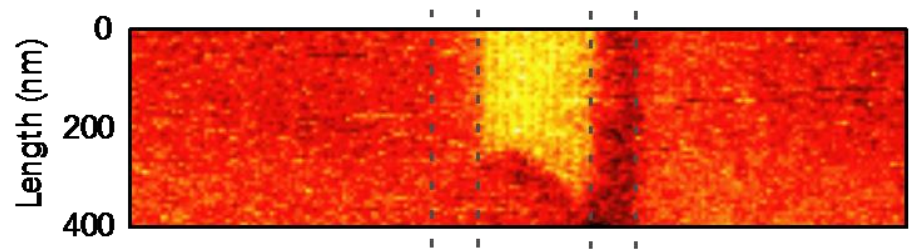

(c)

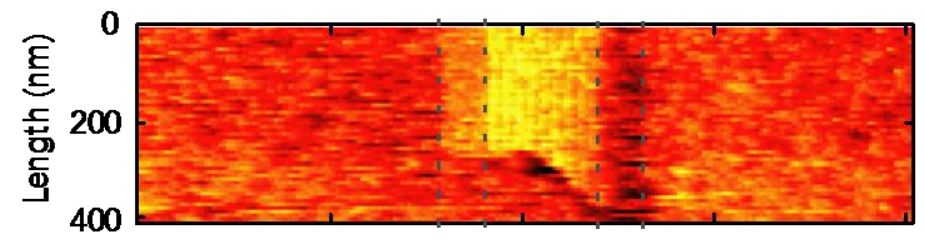

(d)

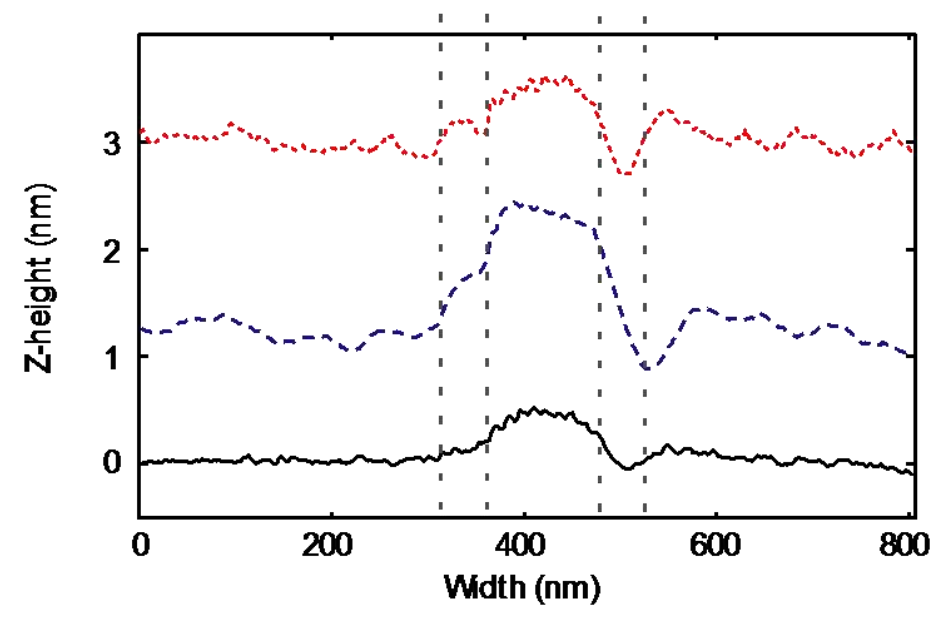


Figure 5:

Modeled change of the tip height as the n-type layer in the BHL undergoes modification of the doping concentration (circles) and the surface state density (crosses), using 1nm tip separation, 10nm tip radius, $0.6 \mathrm{eV}$ contact potential.

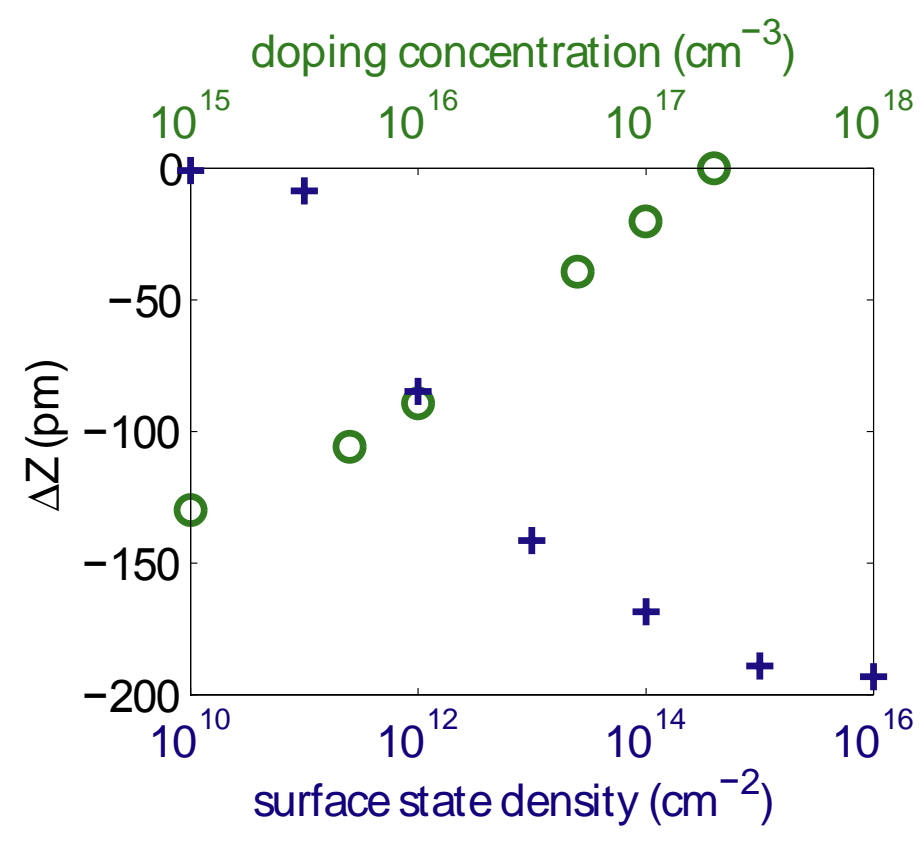

